\title{
IMPACTO DEL TIEMPO NO PRODUCTIVO EN OPERACIONES DE PERFORACIÓN Y ANÁLISIS DE LOS DATOS MEDIANTE LA PRUEBA DE CHICUADRADO
}

Diego Ayala $^{1 *}$, Henry Torres ${ }^{2}$, Raúl Valencia $^{3}$, Marco Loaiza ${ }^{4}$

*A quien debe dirigirse la correspondencia

\begin{abstract}
RESUMEN
El presente estudio se realizó con el objeto de caracterizar la situación del tiempo no productivo NPT en las operaciones de perforación en el oriente ecuatoriano y proponer medidas y acciones que ayuden a mitigar el NPT. Uno de los objetivos de la investigación fue la recolección de datos cuantitativos de los reportes finales de perforación, para los cuales se elaboró una matriz de problemas que permitió organizar y solvente los problemas en estudio, la información se la ordenó por el diámetro de cada etapa del pozo, identificando los problemas predominantes en cada sección y mediante un análisis técnico se determinó la causalidad del NPT. La investigación también se enfocó en obtener el costo-hora asociado al NPT, el estudio se complementó con el análisis Chi cuadrado para comprobar afirmaciones hipotéticas acerca de la relación que puede existir entre las variables que originan NTP, de ésta forma contribuimos a comprender de mejor manera el impacto de los NPT a través de una herramienta matemática.
\end{abstract}

Palabras clave: Tiempo no productivo, Optimización de la perforación, Problemas al perforar.

\section{IMPACT OF NON-PRODUCTIVE TIME (NPT) IN DRILLING OPERATION AND DATA ANALYSIS BY APPLYING THE CHI SQUARE TEST}

\begin{abstract}
The present study was developed aiming at distinguishing the condition of Non Productive Time NPT at drilling operations at Ecuadorian fields and recommending efforts to help decreasing Non Productive Time. The research target was collecting qualitative data from final wellbore reports, for which a classification master chart of problems called matrix was designed that organizes and solves the stuff being studied, the information was arranged according to the diameter corresponding to each section of wellbore identifying in that way the main difficulties into each section and then the cause of NPT was established by applying a technical analysis. The research was focused on obtaining the cost per hour related to NPT also, the study was complemented by adding Chi-square test in order to confirm hypothetical statements about of the relationship that could exist between the variables that cause NPT, in this way the study allows for understanding the impact of NPT through a mathematical resource.
\end{abstract}

Keywords: Non-Productive Time, Drilling Optimization, Drilling problems.

1. Facultad de Ingeniería en Geología y Petróleos. Escuela Politécnica Nacional. PO·Box 17-01-2759. Quito. Ecuador.diego.ayala.t@gmail.com

2. Facultad de Ingeniería en Geología y Petróleos. Escuela Politécnica Nacional. PO·Box 17-01-2759. Quito. Ecuador. fertorrez00@gmail.com

3. Facultad de Ingeniería en Geología y Petróleos. Escuela Politécnica Nacional. PO·Box 17-01-2759. Quito. Ecuador. raul.valencia@epn.edu.ec

4. Enap-Sipetrol PO·Box 17-01-2759. Quito. Ecuador.mloaiza@sipec.com 


\section{INTRODUCCIÓN}

El tiempo requerido para alguna rutina u operación adicional llevada a cabo como resultado de una falla es definido como Tiempo No Productivo o NPT (Rabia, 2002). El NPT en la industria es visto como una medida de cuando las cosas no están dentro de la planificación, cualquier operación fuera del programa de ingeniería y operaciones del pozo debería ser considerado NPT, hay numerosas eventualidades e imponderables que provocan el cese de las operaciones de perforación o que producen un avance marginal en el programa del pozo, todos éstos problemas llevan a un NPT (Ngosi y Mungai, 2014). Esta es la causa principal de los retrasos en los proyectos.

York et al. (2009) encontraron que alrededor del 40\% del NPT fueron causado por inestabilidad del pozo y por la presión de poro (amagos, influjo de aguas poco profundas, pérdida de circulación, la inestabilidad del pozo, derrumbes, y el atascamiento de la tubería).

El presente estudio, es una contribución orientada a enriquecer los modelos utilizados en la planificación para reducir los costos asociados a los NPT, disminuir la incertidumbre, identificar y documentar las fallas de herramientas, optimizar los tiempos y la exactitud para estimar la duración de un proyecto, mejorar la confiabilidad y la integridad operacional de la perforación. Tomando en consideración que no se puede mejorar lo que no se mide (pues todo lo medible es verificable y por ende controlable). Y en este caso no se puede medir sin un apropiado procedimiento del control de calidad in situ (Maindla y Maindra, 2010). Además, Kaiser y Pulshiper (2007) definen que existe factores observables y no observables que impactan los tiempos de perforación de un pozo.

En el análisis realizado se describirán los inconvenientes al perforar, lo que permitirá profundizar en el rendimiento, la optimización del proceso y la eficiencia. Cochener (2010) define la eficiencia como una métrica del rendimiento productivo. Resulta de mucha importancia analizar y encontrar las causas raíces de las operaciones que implican NPT y luego, mejorar dichas operaciones con la implementación de acciones remediales para evitar su recurrencia. Cuando un proyecto de perforación alcanza niveles muy bajos de NPT, se podría decir que se encuentra en la curva de optimización apropiada.

Factores como la planificación, sociabilización en Pre Spud-meeting, la ejecución, la comunicación asertiva, el liderazgo y las destrezas en el gerenciamiento de proyectos, afectarán al rendimiento de la perforación (Marbun, Aristya, Pinem, Ramli y Gadi, 2013). Los Pre Spud-meeting, no deberían destinarse solamente a tratar los planes del proyecto, sino también deberían brindar un espacio a los operadores para obtener sugerencias en la ejecución del trabajo, mano de obra y equipo utilizado (Ramsey, 2007).

\section{RESULTADOS Y DISCUSIÓN}

\subsection{NPT EN OPERACIONES DE PERFORACIÓN}

El NPT total acumulado para el grupo de once pozos considerados es de 92,5 días que corresponde al 19,8\% de un total de 465 días invertidos en la perforación, como se puede observar en la tabla 1 .

La perforación de los pozos direccionales toma en promedio de 15 a 20 días de operaciones, y la perforación de los pozos horizontales toma entre 41 y 78 días de operaciones en la muestra de estudio (Secretaria de Hidrocarburos Ecuador, 2015).

Tabla 1. Detalle general del NPT

\begin{tabular}{cccccc} 
Pozo & $\begin{array}{c}\text { Tipo } \\
\text { pozo }\end{array}$ & $\begin{array}{c}\text { Sección } \\
\text { final } \\
\text { (in) }\end{array}$ & $\begin{array}{c}\text { Total } \\
\text { Días }\end{array}$ & NPT Días & \% NPT \\
\hline 1 & J & $81 / 2$ & 31,36 & 5,19 & 16,54 \\
\hline 2 & J & $81 / 2$ & 43,54 & 5,33 & 12,25 \\
\hline 3 & H & $61 / 8$ & 43,21 & 6,06 & 14,03 \\
\hline 4 & J & $81 / 2$ & 41,00 & 8,31 & 20,27 \\
\hline 5 & J & $81 / 2$ & 26,81 & 1,54 & 5,75 \\
\hline 6 & $\mathrm{H}$ & $61 / 8$ & 41,04 & 3,33 & 8,12 \\
\hline 7 & $\mathrm{~J}$ & $81 / 2$ & 28,02 & 1,56 & 5,58 \\
\hline 8 & $\mathrm{~J}$ & $81 / 2$ & 30,69 & 0,52 & 1,70 \\
\hline 9 & $\mathrm{H}$ & $61 / 8$ & 72,29 & 25,60 & 35,42 \\
\hline 10 & $\mathrm{H}$ & $81 / 2$ & 78,90 & 31,71 & 40,19 \\
\hline 11 & $\mathrm{H}$ & $81 / 2$ & 28,52 & 3,35 & 11,76 \\
\hline & & & 465,39 & 92,52 & 19,88 \\
\hline
\end{tabular}

El NPT por sección del pozo se ilustra en la figura 1, se encuentra que a medida que aumenta la profundidad de perforación también aumenta el NPT, las secciones más problemáticas de perforar son las de 8 1/2" y $12^{1 / 1 / 4}$ ", que juntas reúnen el $75 \%$ de NPT, la sección de 6 $1 / 8$ " presenta un $13,3 \%$ y solo se perfora en pozos horizontales y pozos reentradas. 


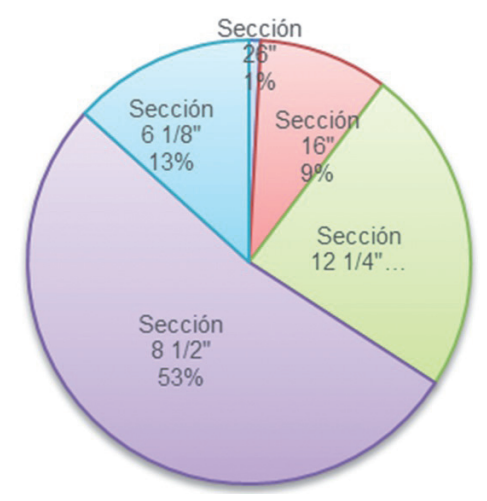

Figura 1. NPT porcentual según la sección. Fuente: Los autores

\subsubsection{NPT EN TRAYECTORIA DIRECCIONAL}

El NPT varía entre 5\% y $20 \%$, el porcentaje más bajo corresponde al pozo 7 con $5,5 \%$, que es el segundo pozo de menor longitud perforada (MD) con trayectoria J. El mayor porcentaje de NPT se presenta en el pozo 4 con un $20,2 \%$, con una trayectoria direccional tipo $\mathrm{J}$.

En 4 de los 7 pozos el porcentaje de NPT supera el 10\% del tiempo total de perforación y en 6 de los 7 pozos supera el 5\%. Los valores se detallan en la figura 2. Las causas de estos NPT, se discuten más adelante en la sección 1.2.1 (causas de los problemas predominantes).

25,0

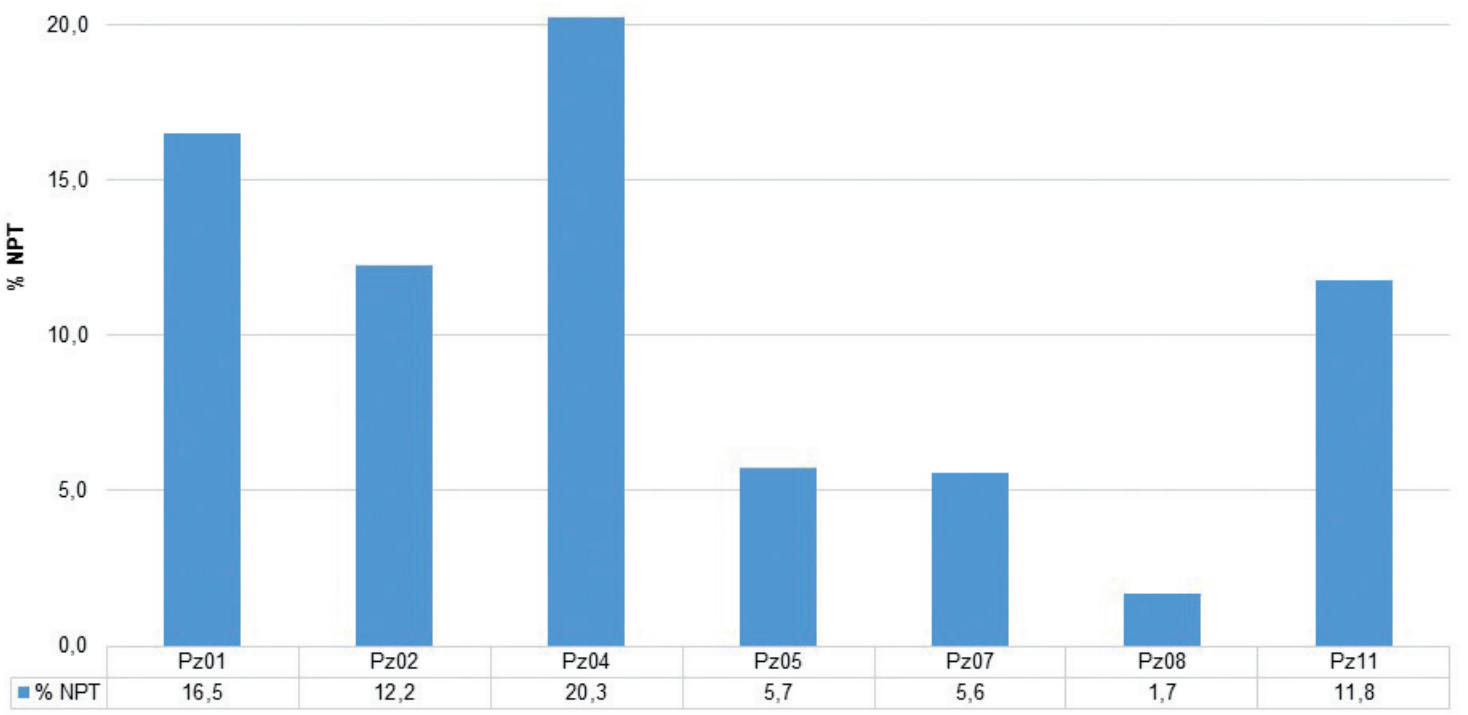

Figura 2. Porcentaje de NPT en pozos direccionales.

Fuente: Los autores

\subsubsection{NPT EN TRAYECTORIA HORIZONTAL}

Los pozos 6 y 3 tienen un porcentaje de NPT de $8 \%$ y $14 \%$ respectivamente, valores que están dentro del rango. Los otros dos pozos presentan porcentajes de NPT bastante elevados de $35,4 \%$ en el caso del pozo 9 y $40,1 \%$ en el caso del pozo 10 . Estos dos pozos son los únicos en los que se presentaron operaciones de desvío o side track. Los valores se detallan en la figura 3. 


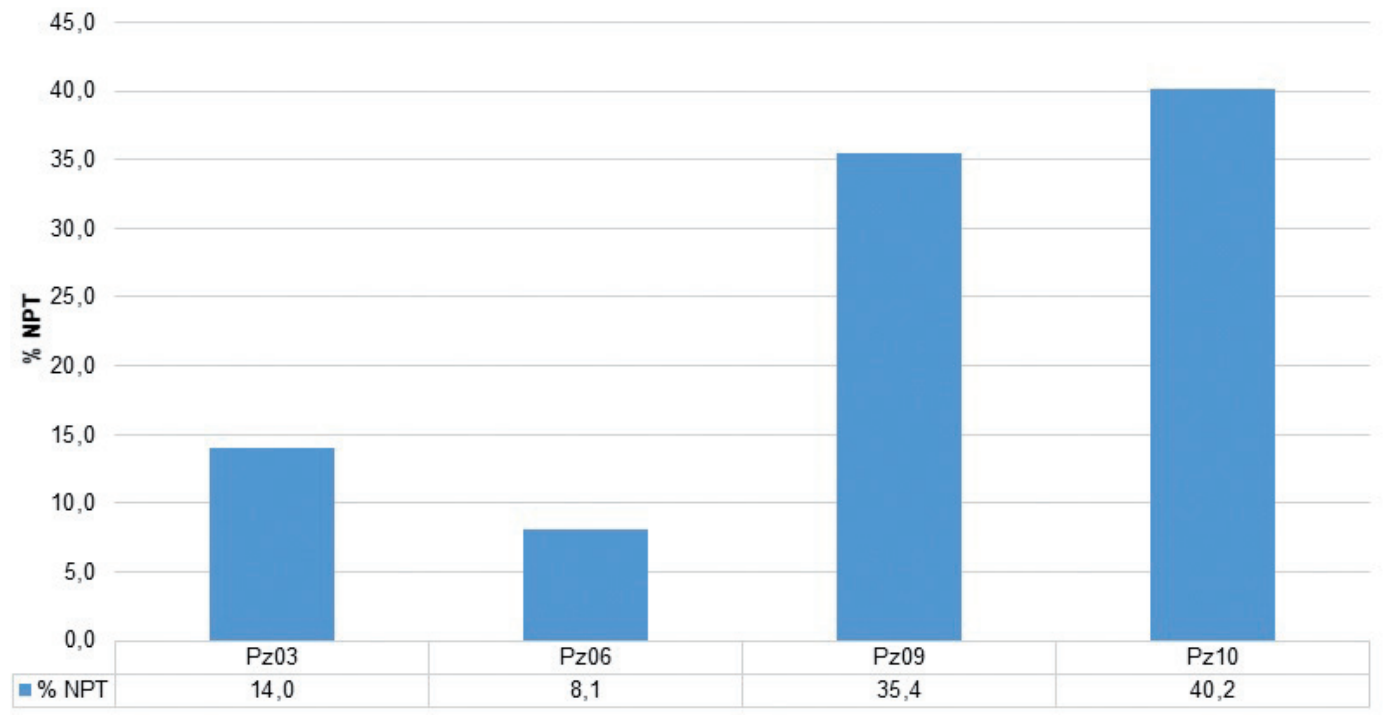

Figura 3. Porcentaje de NPT en pozos horizontales Fuente: Los autores

\subsection{IDENTIFICACIÓNDEPROBLEMAS PREDOMINANTES}

En base de la propuesta de la matriz de NPT (tabla 4, que se detallará más adelante) se elaboró la tabla 2 , en la cual se aprecia que los cuatro primeros problemas son aquellos que ocurren en superficie, los dieciséis restantes ocurren dentro del pozo, la columna Frecuencia indica el número de veces que ocurrió un problema en un mismo pozo, la columna Distribución indica en cuantos de los 11 pozos se encontró un mismo problema. En las secciones superficiales se observó un alto impacto de problemas fuera del pozo y a medida que aumenta la profundidad son los problemas dentro del pozo los que tienen mayor influencia en el NPT.

Tabla 2. Duración, frecuencia y distribución de los NPT

\begin{tabular}{|c|c|c|c|c|c|c|c|c|c|}
\hline & \multirow{2}{*}{$\begin{array}{c}\text { Actividad no } \\
\text { productiva }\end{array}$} & \multicolumn{5}{|c|}{ Sección } & \multirow{2}{*}{ 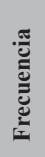 } & \multirow{2}{*}{ 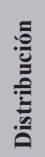 } & \multirow{2}{*}{$\begin{array}{c}\text { Tiempo Tota } \\
\text { NPT } \\
\text { Hrs. }\end{array}$} \\
\hline & & $26^{\prime \prime}$ & $16 "$ & $121 / 4^{\prime \prime}$ & $81 / 2^{\prime \prime}$ & $61 / 8^{\prime \prime}$ & & & \\
\hline \multirow{4}{*}{ 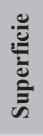 } & Hrs. & 3 & 23 & 20 & 4 & 0 & 50 & 11 & 167 \\
\hline & Problemas TDS & 2 & 5 & 13 & 1 & 0 & 21 & 8 & 112,5 \\
\hline & Clean Flowline/Gumbo & 0 & 3 & 1 & 0 & 0 & 4 & 3 & 14 \\
\hline & Esperas & 0 & 1 & 4 & 2 & 2 & 9 & 5 & 117,5 \\
\hline \multirow{16}{*}{$\underset{\mathscr{E}}{\stackrel{0}{0}}$} & Limpieza y rimado del hoyo & 0 & 1 & 0 & 2 & 0 & 3 & 3 & 2 \\
\hline & Reacondicionar fluido & 0 & 0 & 1 & 0 & 0 & 1 & 1 & 6,5 \\
\hline & Bit balling & 1 & 0 & 0 & 0 & 0 & 1 & 1 & 0,5 \\
\hline & Falla mecánica de la tubería & 0 & 0 & 1 & 2 & 0 & 3 & 1 & 5 \\
\hline & Falla del BHA Herramientas & 0 & 0 & 3 & 2 & 0 & 5 & 4 & 67 \\
\hline & Control Direccional & 0 & 1 & 2 & 1 & 1 & 5 & 4 & 98,5 \\
\hline & Desvío & 0 & 0 & 1 & 2 & 0 & 3 & 2 & 867,5 \\
\hline & Logging Problems & 0 & 0 & 2 & 0 & 0 & 2 & 1 & 4 \\
\hline & Falla de revestimientos & 0 & 2 & 0 & 3 & 0 & 5 & 3 & 81 \\
\hline & Problemas del cabezal & 0 & 1 & 0 & 0 & 0 & 1 & 1 & 0,5 \\
\hline & Problemas de BOP & 0 & 1 & 2 & 0 & 0 & 3 & 2 & 5,5 \\
\hline & Cement problems & 0 & 1 & 0 & 0 & 0 & 1 & 1 & 2 \\
\hline & Pega de tubería & 0 & 0 & 3 & 2 & 0 & 5 & 4 & 66,5 \\
\hline & Pesca & 0 & 0 & 0 & 2 & 1 & 3 & 3 & 120 \\
\hline & Pérdida de circulación & 0 & 0 & 0 & 0 & 0 & 0 & 0 & 0 \\
\hline & Arremetidas & 0 & 2 & 0 & 0 & 0 & 2 & 1 & 4,5 \\
\hline
\end{tabular}




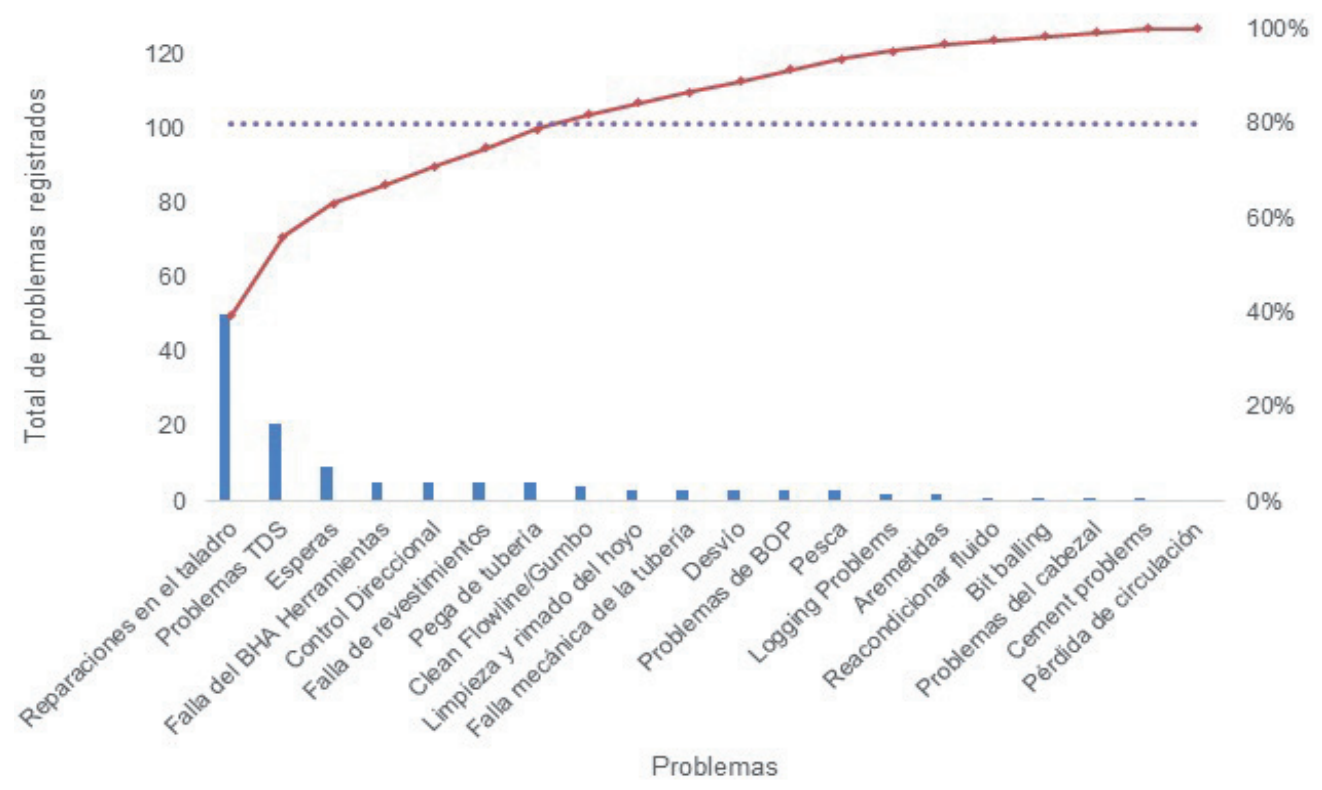

Figura 4. Gráfico de Pareto

Fuente: Los autores

Pareto, figura 4, permite determinar cuáles son las pocas causas que generan la mayor cantidad de defectos. Se basa en que el $80 \%$ de los efectos provienen del 20 $\%$ de las causas (Gutiérrez, 2005). Con la figura 4, se deduce que para eliminar el $80 \%$ de los problemas, hay que determinar las causas que originan las siguientes actividades no productivas: Reparaciones en el taladro, Problemas TDS, Esperas, Falla del BHA herramientas, Control direccional, Falla de revestimientos.
La figura 5, ilustra los tiempos involucrados en la perforación, el NPT, el tiempo productivo y el tiempo total (rojo), el cual da como resultado de la suma de tiempo productivo + NPT. La muestra de 11 pozos se ha ordenado de manera cronológica conforme fueron perforados, siendo el pozo 1 el más antiguo y el pozo 11 el más reciente.

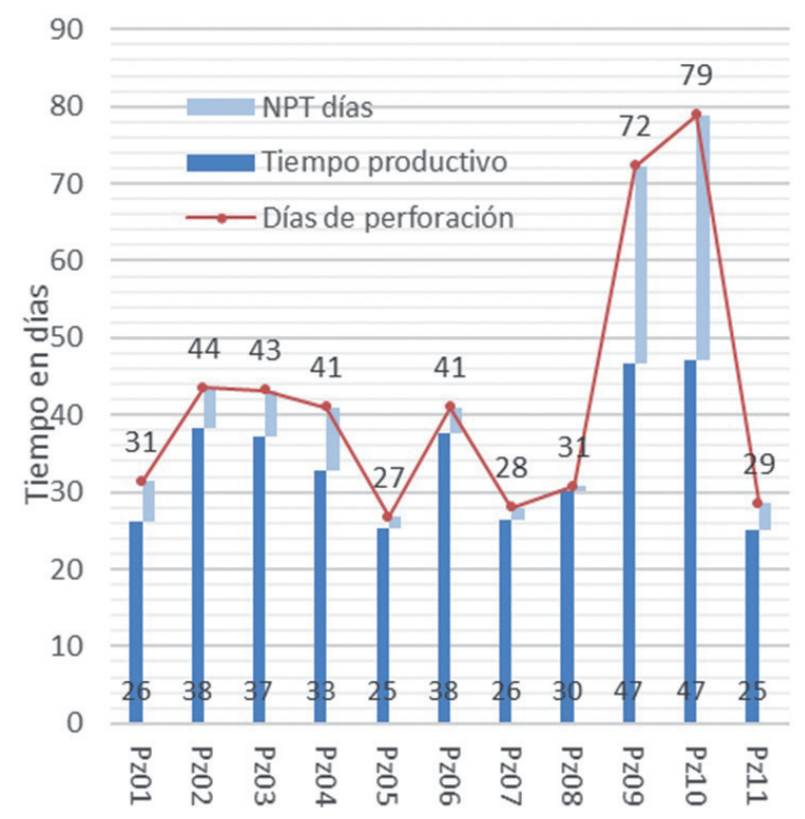

Figura 5. Tiempos involucrados en perforación Fuente: Los autores 


\subsubsection{CAUSAS DE LOS PROBLEMAS PREDOMINANTES}

La tabla 3, registra las condiciones o las operaciones que generan problemas en el pozo; la relación entre causa y problema fue identificada por los técnicos expertos de una manera empírica, indagando en los reportes finales de perforación el detalle de las operaciones, la tabla 3 , permite visualizar los factores que influencian los problemas, lo que permitirá seleccionar y direccionar las medidas a implementar para minimizarlo.

Tabla 3. Causas de los problemas por sección.

\begin{tabular}{|c|c|c|}
\hline \multicolumn{3}{|c|}{ Causas de problemas principales sección de $16 "$} \\
\hline Causas & Problemas & NPT horas \\
\hline $\begin{array}{l}\text { Falta de mantenimiento a equipos; tarjeta electrónica; } \\
\text { presencia de Gumbo }\end{array}$ & $\begin{array}{l}\text { Reparaciones en el taladro; Problemas TDS; Limpieza de } \\
\text { la línea de retorno }\end{array}$ & 121,5 \\
\hline Falla inicial de equipos; inadecuado peso de lodo & Falla de revestimientos; Arremetidas & 6 \\
\hline \multicolumn{3}{|c|}{ Causas de problemas principales sección de 12 1/4" } \\
\hline Causas & Problemas & NPT horas \\
\hline Falta de mantenimiento a equipos; mala logística & Problemas TDS; Reparaciones en el taladro; Esperas & 244 \\
\hline $\begin{array}{l}\text { Falta de mantenimiento a equipos, falla inicial de } \\
\text { equipos, pescado en el pozo }\end{array}$ & $\begin{array}{l}\text { Falla del BHA-Herramientas; Deficiente Control } \\
\text { direccional; Desvío }\end{array}$ & 202 \\
\hline $\begin{array}{l}\text { Circulación y rotación limitada; Falta de mantenimiento } \\
\text { a equipos }\end{array}$ & $\begin{array}{c}\text { Pega o atrapamiento de tubería; deficiente Control } \\
\text { direccional }\end{array}$ & 27,5 \\
\hline \multicolumn{3}{|c|}{ Causas de problemas principales sección de 8 1⁄2” } \\
\hline Causas & Problemas & NPT horas \\
\hline $\begin{array}{l}\text { Falla inicial de equipos; geometría del pozo y } \\
\text { perforación en sobre balance }\end{array}$ & Falla de revestimientos; Pega o atrapamiento de tubería & 129 \\
\hline $\begin{array}{l}\text { Falla inicial de equipos; Pescado; perforación en sobre } \\
\text { balance; Lutitas sensible al agua }\end{array}$ & $\begin{array}{l}\text { Falla de revestimientos; Pesca; Pega o atrapamiento de } \\
\text { tubería; Limpieza y rimado del hoyo }\end{array}$ & 210 \\
\hline Operación de pesca fallida- pega mecánica & Desvío & 746,5 \\
\hline \multicolumn{3}{|c|}{ Causas de problemas principales sección de 6 1/8" } \\
\hline Causas & Problemas & NPT horas \\
\hline $\begin{array}{l}\text { Trayectoria-formación de lutitas- perforación en sobre } \\
\text { balance }\end{array}$ & Pesca & 266,5 \\
\hline
\end{tabular}

Costos variables, son los que dependen del tiempo que toma la perforación de un pozo y son:

- $\quad$ Fluidos de perforación (FP)

- Perforación direccional (PD)

- Control litológico (CL)

- $\quad$ Rol de compañía (RC)

Costos fijos, no dependen del tiempo, están determinados por la parte contractual, por volumen, por listas de precios o tipo Lump Sum y son:

- Brocas

- Cementación

- Otros cargos

- Registros eléctricos

- Tubería de revestimiento

- Cabezal de producción, etc.

La ecuación (1) permite determinar el costo variable (costo / día) de operaciones (Bonilla \& Buestán, 2013):
Solo los costos variables de perforación se verán afectados como resultado de la reducción de los NPT.

La figura 6, refleja los pozos que alcanzaron los costos más bajos asociados al NPT (Pz 4, Pz 5, Pz 8) y los pozos con los costos más altos (Pz2, Pz3, Pz6, Pz10).

Se encontró que, en los reportes de perforación de Schlumberger determinan la variable denominada, costo diario promedio aproximado, valor usado para estimar el costo asociado al NPT, Schlumberger explica en términos generales, como se obtuvo el valor genérico (tomar el dato del costo indicado, remover el costo tangible no relacionado con horas de trabajo, calcular un promedio de costo/hora operación, prorratear la cantidad remante para reflejar el costo del NPT) de 3000 USD / HORA, valor que, es el mismo en cada reporte indistintamente de la geometría del pozo y del taladro contratado. 


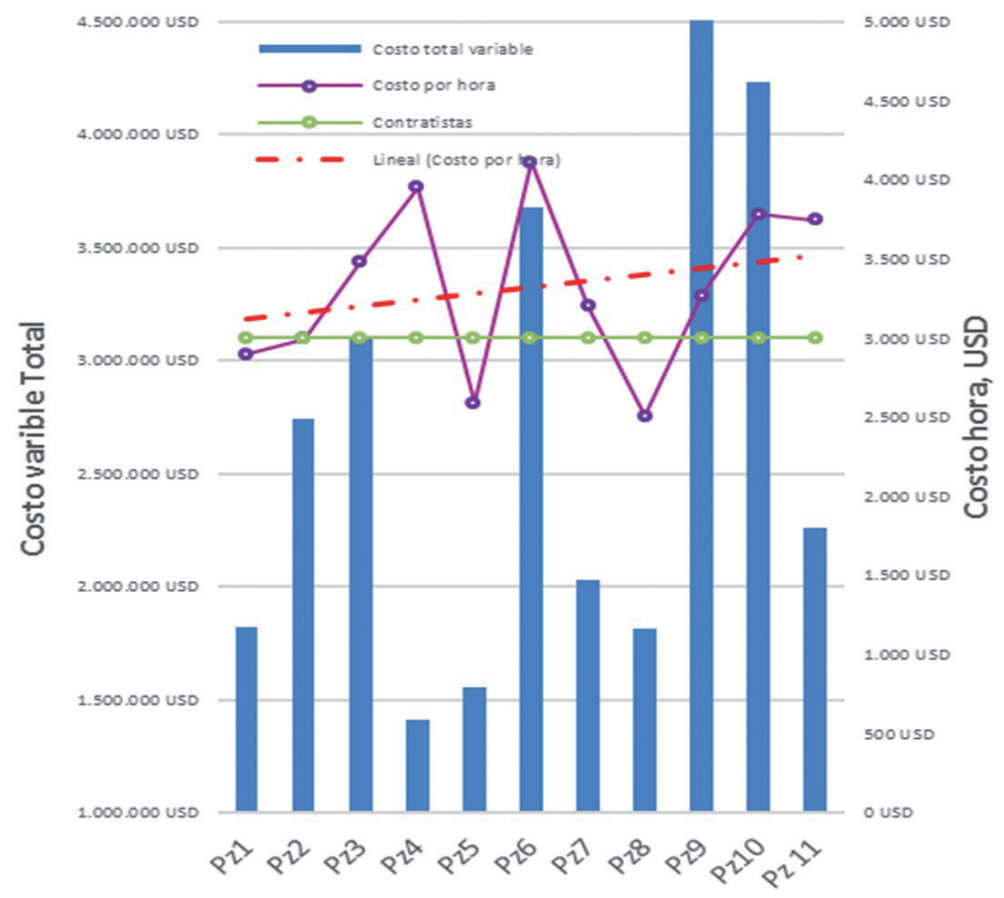

Figura 6. Costo variable de perforación total y por hora.

Fuente: Los autores

En la figura 6, la línea de tendencia (color rojo) representa el valor promedio del costo variable determinado en los 11 pozos, el cual se calculó con la ecuación (1) y es de 3024 USD / HORA, valor que no se aleja del costo estándar estimado por Schlumberger 3000 USD / HORA, Los valores descritos se visualizan en el eje derecho de las ordenadas. En el análisis de los datos se identificó que, el costo/hora varía significativamente al incrementar la complejidad de la geometría del pozo (aumento de TVD, ángulo de desviación y diámetro), el propósito en esta sección es determinar un valor estándar lo más aproximado a la realidad de los precios actuales.

Con el costo/hora, se puede estimar el perjuicio para la operadora por problemas ajenos o no contemplados en la planificación. En los 11 pozos, como se muestra en la tabla 1, el valor acumulado por NPT fue de 92,5 días con un costo asociado de $6.714 .731,52$ USD.

Los problemas de inestabilidad del pozo en operaciones de exploración y perforación cuestan a la industria de la perforación más de 100 millones de dólares por mes y en todo el mundo posiblemente tanto como mil millones de dólares anuales (Moazzeni, Nabaei, \& Azari, 2011)

Reducir los costos de perforación es una de las implicaciones de mejorar la eficiencia (Cochener, 2010).

\subsection{ANÁLISIS DE RESULTADOS}

La información que se dispone sobre NPT se encuentra organizada en diversos formatos, debido a esto no se halla uniformidad y claridad en la identificación de las actividades no productivas, para solucionar e integrar los problemas se propone la matriz estandarizada (Tabla 4).

\subsubsection{CONFIGURACIÓN DE LA MATRIZ}

La matriz permite distinguir, organizar y categorizar los problemas, así como evaluar el tiempo perdido y el costo asociado.

Se configura en filas que muestran los tipos de problemas de perforación y en columnas correspondientes a las secciones del pozo, en las celdas resultantes se registran los NPT incurridos. La columna final de la tabla 4, presenta el total del costo correspondiente a cada problema para el conjunto de pozos.

De arriba hacia abajo la matriz contiene tres bloques bien definidos. Las cuatro primeras filas constituyen el primer bloque, corresponde a los NPT relacionados con problemas del equipo de perforación, equipos de superficie anexos y esperas. 
El segundo bloque, en el área central, muestra los problemas de perforación presentes en las diferentes etapas en la fase perforar, evaluar y revestir el pozo.

En la parte inferior se ubican los problemas frecuentes en la perforación que conforman el tercer bloque, aquellos que se localizan concretamente dentro del pozo.

\subsubsection{VALIDACIÓN DE LOS DATOS}

Los datos tienen que ser congruentes y consistentes entre los valores parciales y totales, en este punto se realiza su verificación, rectificación o eliminación. Para lo cual se comparan los valores registrados en las tablas de NPT, en las curvas de Profundidad vs. Tiempo y en las descripciones de las actividades.

Se descartan los pozos en los que después de una reprogramación (side track -re Entry programado) o desvío, aparentemente no registran más anomalías -NPT.

En el desarrollo del trabajo se encontró que la mayoría de la información sobre NPT registrada en los reportes, no tiende a identificar las causas de los problemas asociados a los NPT, sino más bien a identificar a la línea responsable involucrada en los eventos de NPT, lo que no contribuye realmente a la optimización del proceso de perforación, que sería la finalidad de llevar este tipo de registro.

Para la formulación de una matriz estandarizada es muy importante la contribución de técnicos que posean la experticia en perforación, para que con un criterio objetivo se identifiquen y delimiten correctamente las diversas actividades en el proceso de perforación, a fin de consolidar una matriz de problemas que sea funcional, tarea que no resulta sencilla puesto que el proceso de perforación es bastante dinámico y no se pueden esquematizar rígidamente los tipos de problemas.

Para identificar la causa de los problemas de perforación y el NPT asociado, más allá de las horas acumuladas en eventos fallidos, se consideraron otros factores como la frecuencia y distribución de los problemas, que en conjunto con el tiempo permiten conocer su impacto final en la operación. El procesamiento de la información se encamina para identificar los problemas en cada sección.

El análisis técnico de los problemas predominantes ha sido viable gracias a la matriz de NPT (tabla 4) que, además de llevar un inventario de las actividades no productivas, establece una metodología o protocolo para registrar la información de manera adecuada, a fin de abundar con datos significativos sobre la naturaleza de los problemas presentados y facilitar su análisis.

Se identifica que las secciones más críticas para el proceso de perforación son también las más profundas, hallándose en la sección de 8 1/2" el 52,5\% del NPT y en la de $12 \frac{1 / 4}{4}$ " el 23,8 \% de NPT. También se destaca el porcentaje de NPT en la sección de $61 / 8$ " con el 13,3\% y con la particularidad de que solamente se perfora en tres pozos horizontales.

En los pozos con trayectorias horizontales se identifica que la sección de 8 1/2" representa el 44, $7 \%$ del NPT absoluto, confirmando que el NPT se concentra en las secciones más profundas de pozos con trayectorias horizontales, por lo que éste factor (TVD, diámetro, ángulo de desviación del pozo en relación con la geometría y rigidez del BHA) es importante al analizar las causas de los problemas. (Flores \& Valle, 2014)

Los problemas encontrados en las secciones de 16 " y $12^{1 / 4}$ " son principalmente reparaciones en el taladro y problemas con el TDS, que se acentúan en la sección de $121 / 4$ ", con la presencia de eventos de esperas de destacable duración. Los problemas en la superficie en las secciones de $16^{\text {" y }} 12$ 1/4" representan alrededor de $16,4 \%$ del NPT absoluto, esta evidencia sugiere que a menor profundidad de perforación los problemas en la superficie (reparaciones en el taladro, equipos anexos de superficie y problemas en el TDS) tienen mayor incidencia en el NPT.

El hecho de determinar los problemas predominantes asociados con el NPT no es suficiente para conocer sus causalidades, por eso, al llevar a cabo el análisis técnico se descubre indirectamente que los problemas obedecen a ciertas naturalezas independientemente del tipo de problema.

Se introdujeron previamente los criterios de NPT "Perdido" y NPT "Problemas", pero con el desarrollo del trabajo se consigue ampliar sus nociones indicando que el NPT "Perdido" corresponde a fallos internos del proceso que no dependen de las condiciones activas del pozo, es decir contiene al NPT por fallos en la superficie y el NPT "Problemas" corresponde a las anomalías afectadas por la complejidad que representa las condiciones de la formación y el pozo, es decir contempla al NPT por problemas en el hoyo. 
Como resultado, se ha profundizado en mayor grado sobre las causas del NPT a diferencia de las otras referencias bibliográficas consultadas para el desarrollo de este trabajo.

En la sección de 12 1/4", existe una contribución de problemas dentro del pozo, sin embargo al analizar los inconvenientes se encuentra que estos problemas reflejados en el fondo son propiciados en la superficie. Aquí las causas de los problemas son los fallos en superficie $(50 \%)$ y otro alto porcentaje son problemas inducidos $(>40 \%)$ y solo una pequeña porción se originan dentro del pozo por dificultades con el control direccional y atrapamiento o pega de tubería.

En la sección de $81 \frac{1}{2}$, se evidencia lo crítica que se vuelve la operación con trayectorias horizontales en las formaciones Napo (formación del cretácico, secuencia de lutitas fisiles reactivas inestables, calizas y areniscas, con presencia de crudo) y Hollín (formación del pre cretácico de arenisca cuarzosa blancas, formación estructuralmente frágil e inconsolidada, con presencia de crudo). El NPT se ve agravado por la combinación de las dificultades regionales de la formación con las trayectorias y la no utilización de buenas prácticas operacionales.

Las maniobras con la tubería dentro del pozo en las secciones de $81 / 2$ " y $61 / 8$ ", son operaciones especialmente críticas, que derivan en pegas mecánicas y herramientas atrapadas que generan pescas, los cuales se convertirán en los NPT más prolongados debido a las operaciones de desvío que se deben efectuar. Hablando de la sección de $81 / 2 "$, se considera que el 11,9\% del NPT sería atribuible a problemas inducidos, el 19,3\% del NPT está relacionado con dificultades en el pozo y la formación y un $68,8 \%$ de NPT además se encuentra influenciado por la trayectoria de los pozos.

Los pronósticos geomecánicos y la aplicación del control predictivo han dado buenos resultados, por lo que se recomienda evaluarlos para saber si ameritan continuar y mejorar.

Tabla 4. Matriz de los problemas que generan NPT

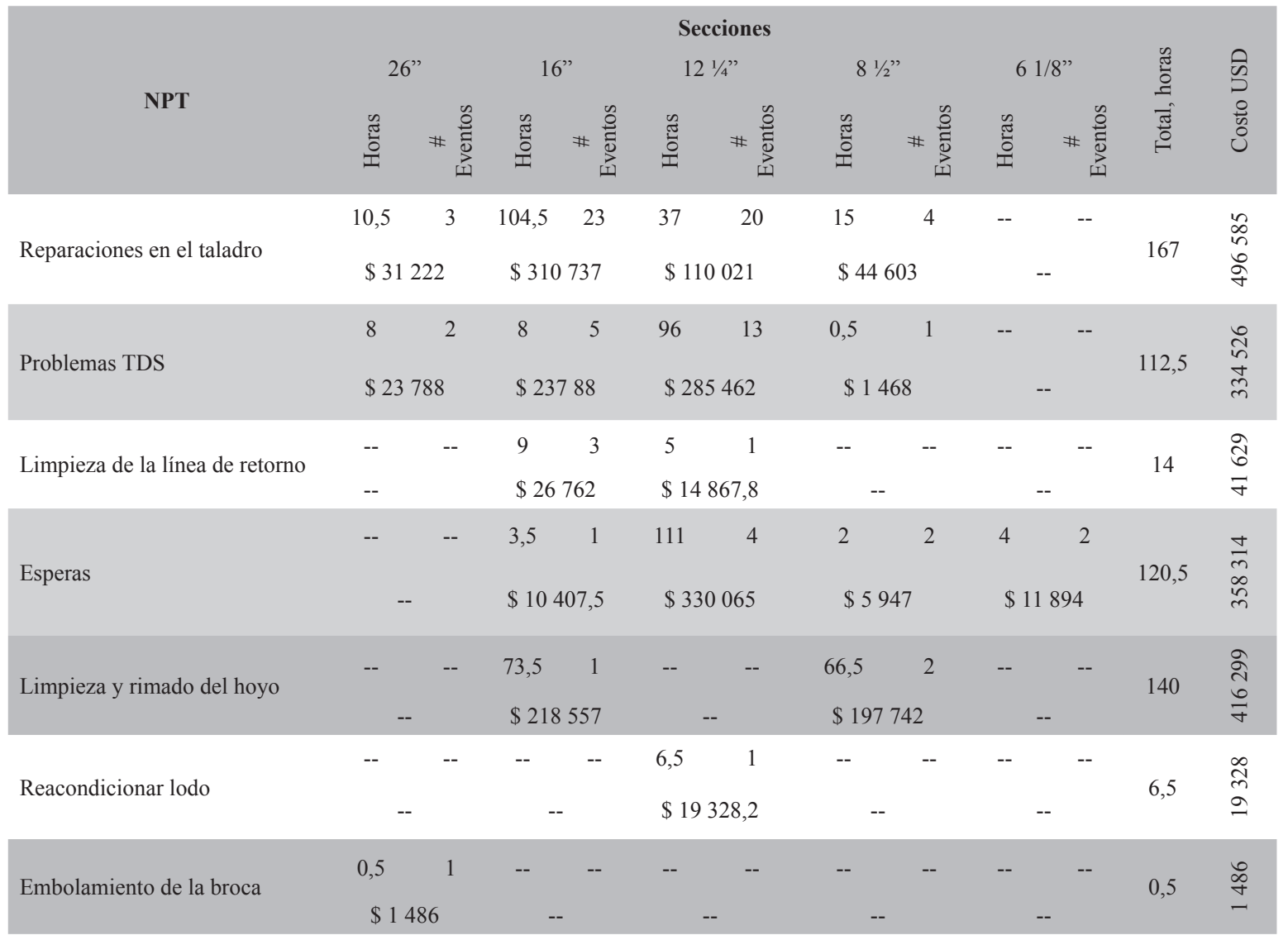




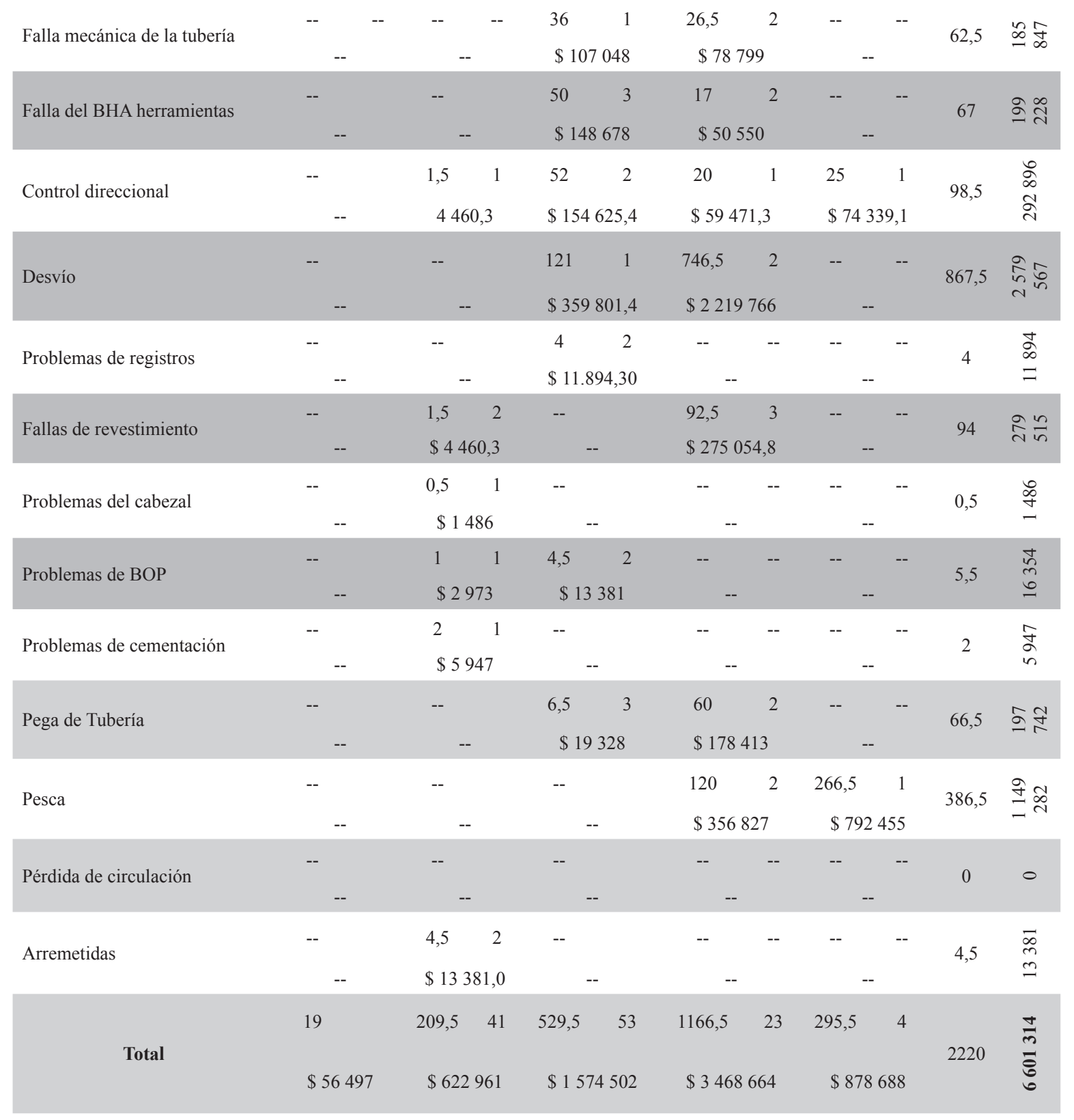

\subsection{ANÁLISIS DE LOS PROBLEMAS EN EL POZO MEDIANTE CHI CUADRADO}

Chi cuadrado permite estudiar la co-relación que existe entre dos variables cualitativas mediante la elaboración de tablas de contingencia (Fahim, 2007). A continuación, se detallan los pasos realizados en el análisis:

1. Formular una Hipótesis Teóricas () por cada interrogante que se desea plantear. Se debe tomar en cuenta que las dos variables en estudio son independientes, pero el método de Chi cuadrado buscará la evidencia que supongan su interdependencia.

2. Para contrastar la $H_{0}$ :

2.1 Ubicar de manera ordenada y de forma lógica las frecuencias observadas (fe)

2.2 Calcular las frecuencias marginales de fila.

2.3 Calcular las frecuencias marginales de columna.

2.4 Para contrastar la $H_{0}$, se debe determinar las frecuencias esperadas así: 


$$
F e_{i j}=\frac{(\text { Total fila } I-\text { ésima })(\text { Total Columna } j-\text { ésima })}{\text { Total Global }}
$$

3. Con los valores determinados se elabora una tabla de frecuencias esperadas.

4. Medir las discrepancias y utilizar la expresión estadística estadígrafo de contraste:

$$
x^{2}=\sum \frac{\left(f o_{i j}-f e_{i j}\right)}{f e_{i j}}
$$

Donde:

$f o_{i j}=$ frecuencia observada para la ij- ésima casilla.

$f e_{i j}=$ frecuencia observada para la ij- ésima casilla.

5. Si la Hipótesis nula es cierta, sigue una distribución Chi cuadrada con:

$$
\text { Grados de libertad }=(i-1)(j-1)
$$

Dónde: $\mathrm{i}$ = número de filas; $\mathrm{j}$ número de columnas

6. La hipótesis nula $\left(H_{0}\right)$ se rechaza, es decir se acepta la dependencia entre variables cuando:

$$
. \mathrm{X}^{2} \text { experimental }>\mathrm{x}^{2} \text { crítico }
$$

7. En la tabla de Chi cuadrado se identifica el valor de intersección dado por el valor de Grados de Libertad y el valor de Nivel de Riesgo 0,05.

8. El valor teórico que proporciona este modelo tiene relación con los Grados de Libertad y con la dimensión de la tabla en estudio.

9. Comparar el valor calculado experimental con el valor teórico para obtener un criterio que fundamente la hipótesis:

- La respuesta depende del tratamiento Si:

$$
\mathrm{x}_{0.05 \text {,grados de libertad }}^{2} \text { (teórico) }>x^{2}(\text { experimental })
$$

- La respuesta No está relacionada con el tratamiento:

$$
\mathrm{x}_{0.05 \text {,grados de libertad }}^{2} \text { (teórico) }<x^{2} \text { (experimental) }
$$

(Kreige \& Meriam, 2007)

\subsubsection{CHI CUADRADO TEST}

1. Formulación de las hipótesis de interés.

1.1 A la primera hipótesis, se la denominará hipótesis (a), busca encontrar una relación entre los problemas que son recurrentes en un mismo pozo y la ocurrencia de esos problemas dentro de la muestra de 11 pozos:

HIPÓTESIS NULA: No existe dependencia entre problema local y problema regional, con el $95 \%$ de confiabilidad

1.2 A la segunda hipótesis se la denominará hipótesis (b), trata de buscar una correlación entre los problemas identificados en superficie con los problemas en el fondo del pozo.

HIPÓTESIS NULA: No existe dependencia entre problemas de superficie y los de fondo, con el $95 \%$ de confiabilidad.

2. Calcular las frecuencias esperadas en función de la tabla 2 para la hipótesis (a) e hipótesis (b), como se muestra en la tabla 5 y tabla 6 .

El significado de los términos utilizados para tabular los datos de frecuencia en la tabla 5 y tabla 6 , a continuación:

Local, es la frecuencia con la que ocurre un problema en las diferentes secciones de un mismo pozo.

Regional, es la frecuencia con la que ocurre un problema en todo el espacio muestral, en las diferentes secciones de los pozos en estudio. 
Tabla 5. Frecuencias esperadas para hipótesis (a)

\section{FRECUENCIA ESPERADA}

Todos los pozos y todas las secciones

\begin{tabular}{|c|c|c|c|}
\hline & Actividad no productiva & Local & Regional \\
\hline \multirow{4}{*}{ 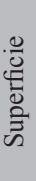 } & Reparaciones en el taladro & 41,65 & 19,35 \\
\hline & Problemas TDS & 19,80 & 9,20 \\
\hline & Clean Flowline/Gumbo & 4,78 & 2,22 \\
\hline & Esperas & 9,56 & 4,44 \\
\hline \multirow{15}{*}{$\begin{array}{l}\text { 을 } \\
\text { 오 }\end{array}$} & Limpieza y rimado del hoyo & 4,10 & 1,90 \\
\hline & Reacondicionar fluido & 1,37 & 0,63 \\
\hline & Bit balling & 1,37 & 0,63 \\
\hline & Falla mecánica de la tubería & 2,73 & 1,27 \\
\hline & Falla del BHA Herramientas & 6,15 & 2,85 \\
\hline & Control Direccional & 6,15 & 2,85 \\
\hline & Desvío & 3,41 & 1,59 \\
\hline & Logging Problems & 2,05 & 0,95 \\
\hline & Falla de revestimientos & 5,46 & 2,54 \\
\hline & Problemas del cabezal & 1,37 & 0,63 \\
\hline & Problemas de BOP & 3,41 & 1,59 \\
\hline & Cement problems & 1,37 & 0,63 \\
\hline & Pega de tubería & 6,15 & 2,85 \\
\hline & Pesca & 4,10 & 1,90 \\
\hline & Arremetidas & 2,05 & 0,95 \\
\hline
\end{tabular}

La tabla 5, describe las frecuencias para la hipótesis (a).

Tabla 6. Frecuencias observadas y esperadas para hipótesis (b)

\begin{tabular}{|c|c|c|c|}
\hline \multicolumn{4}{|c|}{ FRECUENCIA OBSERVADA } \\
\hline D & Local & Regional & Total \\
\hline Problema & \multicolumn{3}{|c|}{ Todas las secciones todos los pozos } \\
\hline Superficie & 84 & 27 & 111 \\
\hline Fondo & 43 & 75 & 118 \\
\hline Total general & 127 & 102 & 229 \\
\hline \multicolumn{4}{|c|}{ FRECUENCIA ESPERADA } \\
\hline D & Local & Regional & Total \\
\hline Proviena & \multicolumn{3}{|c|}{ Todas las secciones todos los pozos } \\
\hline Superficie & 61 & 49 & - \\
\hline Fondo & 65 & 52 & - \\
\hline
\end{tabular}

La tabla 6, describe las frecuencias para la hipótesis (b). Se determinará con la ecuación (3) el estadístico de prueba Chi-cuadrado, que compara las frecuencias que entregan los datos de la muestra (frecuencias observadas) con las frecuencias esperadas.

a) Para hipótesis (a)

El valor de prueba $x^{2}=3,16413162$
Este valor del estadístico de prueba, se lo comparará con un valor de la tabla de probabilidades para Chi-cuadrado $\left(x^{2}\right)$. Esta tabla contiene solo valores positivos porque Chi-cuadrado sólo da resultados positivos.

b) Para la hipótesis (b)

El valor de prueba $x^{2}=2,0348575$

3. Uso de la tabla de Chi Cuadrado

Se obtendrá los grados de libertad con la ecuación (4).

a) Para la hipótesis (a)

Los grados de libertad $=18$

b) Para la hipótesis (b)

Los grados de libertad $=1$

4. Tabla de Chi cuadrado

a) Hipótesis (a), se elige un nivel de significación alfa $=0,05$. Entonces un valor de tabla para $\mathbf{x}^{2}$ asociado a 18 grados de libertad y alfa 0,05 es 11,64 .

b) Hipótesis (b), se elige un nivel de significación alfa $=0,05$. Entonces un valor de tabla para $\mathbf{x}^{2}$ asociado a 1 grados de libertad y alfa 0,05 es 35,64 .

\subsubsection{ANÁLISIS DE LA FIGURA}

Para la hipótesis (a):

En la figura 7, se observa que 11,64 se encuentra a la izquierda de valor $\mathbf{P}=\mathbf{0 , 8 6 5}$, la probabilidad asociada a valores superiores a 11,64 es mayor que alfa $(0,05)$.

Según esto, se acepta la hipótesis nula: No existe dependencia entre problema local y problema regional, con el $95 \%$ de confiabilidad.

Para la hipótesis (b):

En la figura 7, se observa que 35,64 se encuentra a la derecha de valor $\mathbf{P}=\mathbf{2 , 3 E - 0 9}$, la probabilidad asociada a valores superiores a 35,64 es menor que alfa $(0,05)$.

Según esto, se acepta la hipótesis alterna: Si existe dependencia entre problemas de superficie y los de fondo, con el $95 \%$ de confiabilidad. 


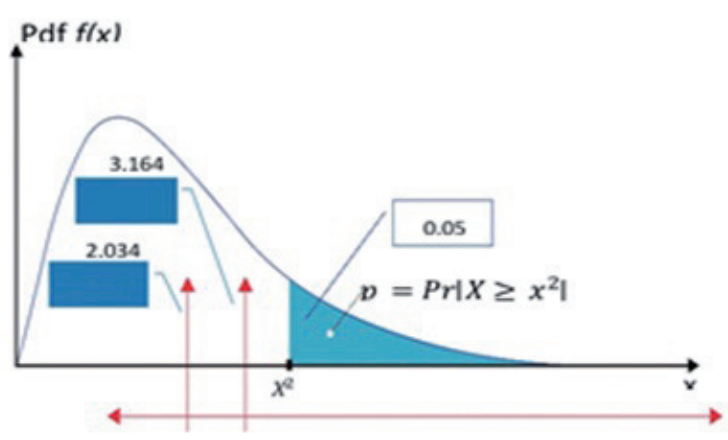

Figura 7. Análisis de las hipótesis según el grado de significancia

Fuente: Los autores

\section{CONCLUSIONES}

En las secciones de 16 " y $12 \frac{1 / 4}{4}$, existe un alto impacto (hasta un $50 \%$ ) del NPT generado por los problemas en superficie; adicionalmente la sección de $81 \frac{1}{2}$ ", es la más crítica y el NPT está influenciado por las dificultades de la cuenca amazónica del Ecuador, que se manifiestan en las zonas de lutitas en Napo.

El 31\% del NPT es altamente controlable en el proceso de perforación, está dado básicamente por factores internos de la operación, falencias operativas y administrativas, baja percepción del riesgo.

El $57 \%$ del NPT corresponde a problemas de alta complejidad, de los cuales el $11 \%$ está relacionado a las condiciones intrínsecas hoyo-formación y el 46 \% está influenciado por el tipo de trayectoria.

Se logró inferir que el costo variable de perforación actual es de 3024 USD/HORA, y el valor acumulado asociado al NPT en la muestra de 11 pozos es de 6.714.731 USD.

Al aplicar el Test de Chi cuadrado a los datos se determinó que, los problemas no guardan una relación entre sí; pero se establece que existe una correlación entre los problemas de superficie y la ocurrencia de problemas en el fondo del pozo.

\section{RECOMENDACIONES}

Implementar una matriz que estandarice las categorías de NPT, de forma que sean mutuamente excluyentes, que permita identificar claramente al problema y que sea adoptada en todos los reportes de perforación.
Realizar una inspección nivel 1 del taladro antes de iniciar los trabajos de perforación en un nuevo pozo.

Desarrollar una investigación técnica acerca de los NPT generados por desvíos del pozo, para determinar la causa raíz de este tipo de inconvenientes y con éstos resultados realizar una matriz de riesgo operativa de las diferentes líneas de servicio incluido el taladro de perforación.

Mejorar el diseño del BHA (utilizar RSS, Rotable Stereeable System), con lo cual siempre el BHA estará en modo rotación, eliminando la necesidad de realizar slides (deslizamientos), con el fin de corregir las trayectorias y a su vez minimizando la probabilidad de tener pegas y/o atrapamientos de tubería al perforar las secciones profundas de $81 / 2$ " y $61 / 8$ " por la presencia de formaciones con distinto contraste de densidad, que muchas veces generan washouts aumentando el riesgo de tener incidentes con pérdida de herramientas.

Extender y mejorar la aplicación del control predictivo para tomar decisiones adecuadas y a tiempo, que permitan reducir el impacto de las anomalías y complejidades ofrecidas por las formaciones al proceso de perforación.

Evaluar los estudios geomecánicos, puesto que los problemas generados dentro de las formaciones repercuten en un $11 \%$ del NPT, lo que sugiere una aplicación aceptable de estos estudios, como por ejemplo generar el tren de densidad de lodos, mantener el hoyo en condiciones óptimas y diseñar trayectorias direccionales que sean perpendiculares al rumbo de esfuerzos.

\section{AGRADECIMIENTO}

Agradecemos al ingeniero Marco Loaiza por la asesoría brindada al realizar la revisión técnica del artículo.

\section{REFERENCIAS}

1. Bonilla, M., \& Buestán, A. (2013). Estudio del Límite Técnico para la Perforación de Pozos en los Campos Cuyabeno y VHR. Quito.

2. Cochener, J. (2010). Quantifying Drilling Efficiency. U.S. Energy Information Administration, 16.

3. Fahim, M. (2007). Chi-Squared test of independence. Calgary: University of Calgary. 
4. Flores, P., \& Valle, D. (2014). Propuesta para Optimizar las Operaciones de Perforación de 9 Pozos en el Campo Amistad, Mediante el Análisis de los Problemas Presentados en la Perforación de los Pozos Amistad 5 y 7 del Golfo de Guayaquil Bloque 3. Quito.

5. Gutiérrez, A. (2005). Aplicación de los Círculos de Calidad de una Organización. Pachuca.

6. Kaiser, M., \& Pulshiper, A. (2007). Generalized Fuctional Models for Drilling Cost Estimation. SPE.

7. Kreige, G., \& Meriam, J. (2007). Engineering Mechanic Statics. John Wiley \& Sons, Inc.

8. Loaiza, M., Ramírez, F., Morales, D., Colmenares, E., \& Tapia, E. (2015). Techniques and Benefits of Drilling into 2 Sections in the Ecuadorian Basin. SPE, 8.

9. Maindla, E., \& Maindra, W. (2010). Rigorous Drilling Nonproductive-Time Determination and Elimination of Invisible Lost Time: Theory and Case Histories. SPE, 9.

10. Marbun, B., Aristya, R., Pinem, R., Ramli, B., \& Gadi, K. (2013). Evaluation of Non Productive Time of Geothermal Drilling Operations - Case Study in Indonesia. Stanford University, 9.

11. Moazzeni, A., Nabaei, M., \& Azari, A. (2011). Reducing Consumed Energy while Drilling an Oil Well through a Deep. Omidihe Iran: Advanced in Petroelum Exploration and Development.
12. Ngosi, R., \& Mungai, K. (2014). Analysis of Nonproductive Time in Geothermal Drilling - Case Study Menengai Field in Kenya. African Rift Geothermal Conference, 14.

13. Rabia, H. (2002). Well engineering and construction.

14. Ramsey, M. (2007). Improved Drilling Technical Training and Communications for Effective Rig Utilization and Accelerated Promotional Schedules During the Big Crew Change and Associated. Houston.

15. Secretaria de Hidrocarburos Ecuador. (2015) Datos Abiertos.

16. York, P., Panitchard, D., Dodson, J., Dodson, T., Rosenberg, S., Gala, D., \& Utama, B. (2009). Eliminating Non-Productive Time Associated with Drilling Trouble Zones. Offshore Technology Conference OTC, 18.

\section{ABREVIATURAS}

$$
\begin{array}{ll}
\text { BHA } & =\text { Ensamblaje de fondo } \\
\text { BOP } & =\text { Preventor de reventones } \\
\text { CDP } & =\text { Costo por día } \\
\text { H } & =\text { Pozo horizontal } \\
\mathrm{J} & =\text { Pozo tipo J } \\
\text { NPT } & =\text { Tiempo no productivo } \\
\text { MD } & =\text { Profundidad medida } \\
\text { TDS } & =\text { Sistema top drive } \\
\text { TVD } & =\text { Profundidad vertical verdadera }
\end{array}
$$

